\title{
Validity and Reliability of the Thai Version of the Pediatric Obstructive Sleep Apnea Screening Tool
}

\author{
Archwin Tanphaichitr ${ }^{1}$, Pitchayanan Chuenchod ${ }^{2}$, Kitirat Ungkanont ${ }^{1}$, Wish Banhiran ${ }^{1}$, \\ Vannipa Vathanophas ${ }^{1}$, and David Gozal ${ }^{3}$ \\ ${ }^{1}$ Mahidol University Faculty of Medicine Siriraj Hospital \\ ${ }^{2}$ Koh Samui Hospital \\ ${ }^{3}$ University of Missouri School of Medicine
}

February 21, 2021

\begin{abstract}
Objective: Obstructive sleep apnea (OSA) is highly prevalent in children and requires an expensive and relatively unavailable sleep study for diagnosis. This study was undertaken to translate the previously validated OSA screening tool (POSAST) to the Thai language and assess its accuracy and test-retest reliability in at-risk symptomatic children. Study design: Prospective cross-sectional cohort study Methods: Pediatric patients clinically referred for suspected OSA who underwent overnight polysomnography (PSG) were recruited, and caregivers completed the Thai version of the POSAST. The same questionnaire was completed again after 2-4 weeks. Results: One hundred and ten subjects completed the study. The mean age was $8.4 \pm 2.9$ years. The mean apnea-hypopnea index (AHI) was $10.9 \pm 11.9$ events/hour. Test-retest reliability (Pearson correlation coefficient $=0.96, \mathrm{P}<0.001)$ and internal consistency (Cronbach's alpha coefficient $=0.82, \mathrm{P}<0.001$ ) between each question were excellent. A cumulative equation-derived score cut-off of 1.9 yielded $78.4 \%$ sensitivity and $50.0 \%$ specificity, while a numerical additive score cut-off of 8 corresponded to $81.1 \%$ sensitivity and $52.8 \%$ specificity for diagnosing moderate and severe OSA (AHI [?] 5 events per hour) Conclusion: The internal consistency and reproducibility of the Thai version of the POSAST are satisfactory, display acceptable validity, and the instrument can be used for screening symptomatic Thai children for OSA.
\end{abstract}

\section{Hosted file}

dg Validity and Reliability of Thai Version 18.2.2021.pdf available at https://authorea. com/users/397023/articles/510063-validity-and-reliability-of-the-thai-version-of-thepediatric-obstructive-sleep-apnea-screening-tool

\section{Hosted file}

Table1-5 (16.2.2021).pdf available at https://authorea.com/users/397023/articles/510063validity-and-reliability-of-the-thai-version-of-the-pediatric-obstructive-sleep-apneascreening-tool 

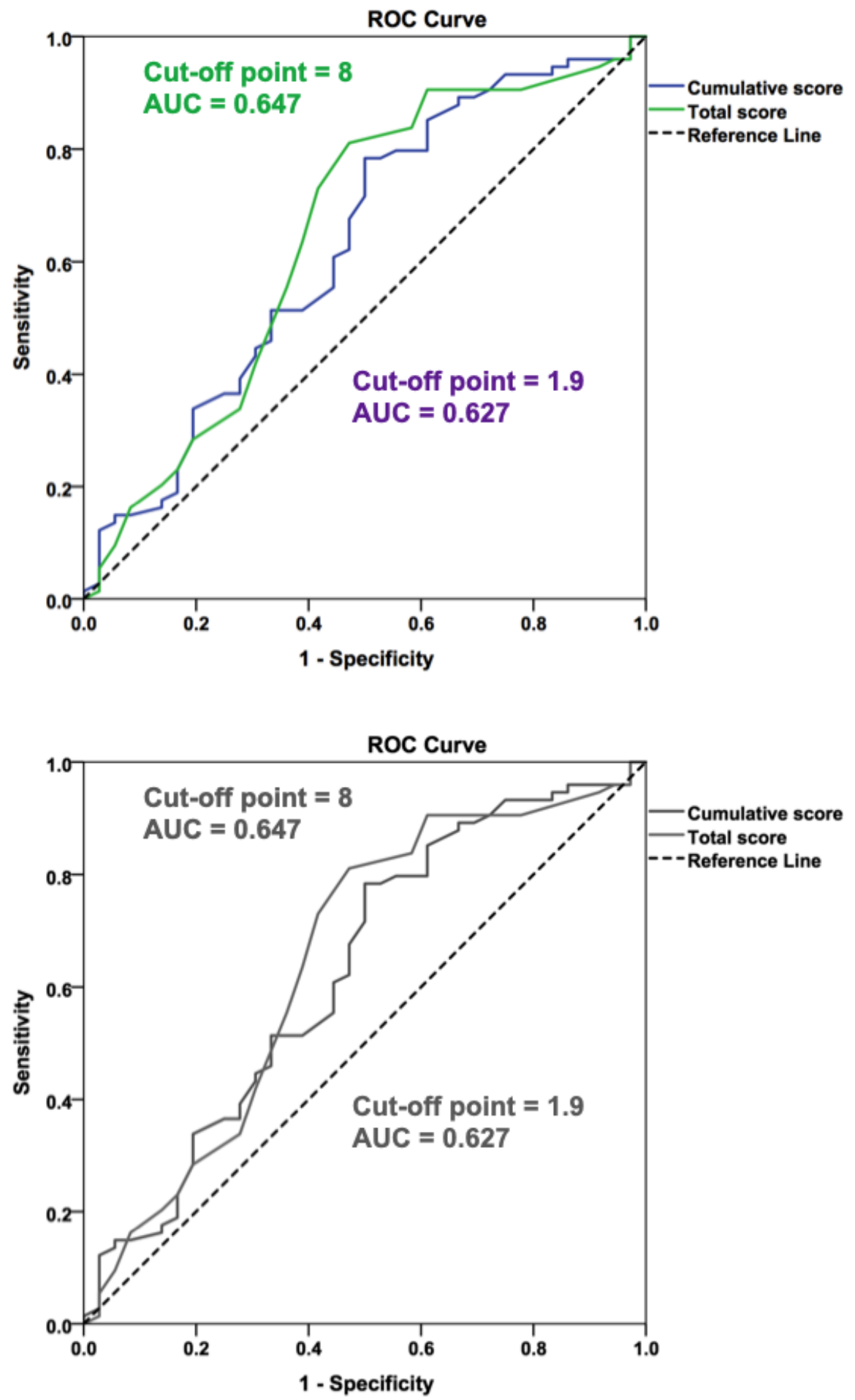